\title{
Problemas de conducta en niños víctimas de violencia familiar: reporte de profesores ${ }^{1}$
}

\author{
Martha Frías Armenta \\ Blanca Fraijo Sing \\ Nehemias Cuamba Osorio \\ Universidad de Sonora (México)
}

\begin{abstract}
Resumen
El objetivo de esta investigación fue analizar la relación de los problemas de conducta que los niños presentan en la escuela y el maltrato infantil. La muestra la constituyeron 110 menores; 61 fueron identificados como maltratados y 50 fueron de la población general. Se aplicó la lista de chequeo de Achenbach (Achenbach, 1991; Achenbach \& McConaughy, 1997) a los maestros, así como la Escala de Tácticas de Conflicto de Straus et al. (1998), la de Depresión de Hamilton (1959), obteniendo también variables demográficas de los niños. Se probó un modelo de ecuaciones estructurales en donde la variable dependiente fueron los problemas de conducta y las independientes la violencia familiar y la depresión. Los resultados indican que el maltrato tuvo un efecto significativo en los problemas de conducta de los menores, mediada por la depresión. Concluimos que los profesores deben ser entrenados en la detección y atención del maltrato infantil para prevenir problemas sociales graves como la delincuencia.
\end{abstract}

Descriptores: violencia familiar; problemas de conducta; reporte de profesores; niños

\begin{abstract}
Conduct problems in young victims of family abuse: teachers' report. The objective of this study was to analyze the relationship between conduct problems that children display at school and child abuse. The sample was constituted by 110 minors; 61 were identified as abused and 50 were from the general population. Achenbach's checklist (Achenbach, 1991; Achenbach \& McConaughy, 1997) was administered to teachers, and Straus et al.'s (1998) Tactics of Conflict Scale and Hamilton's (1959) Depression Scale were administered to children. Demographic variables were also considered. A structural equations model was tested wherein the dependent variable was conduct problems, while family violence and depression were the independent variables. Results indicated that child abuse had an indirect effect on children's conduct problems, which was mediated by depression. We concluded that teachers should be trained in detecting and attending child abuse in order to prevent further serious social problems such as delinquency.
\end{abstract}

Keywords: family violence; conduct problems; teachers' reports; children

$\mathrm{L}$ a violencia familiar es definida por la Organización Mundial de la Salud (2003) como "todo acto de violencia que tiene como resultado posible o real un daño físico, sexual o psicológico, incluidas las amenazas, la coerción o la privación arbitraria de la libertad, ya sea que ocurra en la vida pública o en la vida privada". Abarca, sin carácter limitativo, la violencia física, sexual y psicológica en la familia y ésta puede dirigirse hacia la pareja, los ancianos o los niños. La violencia hacia los niños se denomina abuso o maltrato infantil. Este mismo organismo define el maltrato infantil como

cualquier forma de daño físico y/o emocional, abuso sexual, negligencia o cualquier forma de trato negligente, comercial $u$ explotación, que resulta en el daño actual o potencial a la salud, sobrevivencia o desarrollo de la dignidad, en el contexto de una relación de responsabilidad, confianza o poder. (World Health Organization, 1999, pp. 15-16) 
En México la violencia familiar ha aumentado considerablemente en los últimos años. En el Estado de Sonora (norte del país), por ejemplo, el 49.8\% de las mujeres han sufrido al menos un incidente de violencia en los últimos 12 meses (Instituto Nacional de Geografía e Informática, 2006). Este porcentaje es el más alto de la república Mexicana. En general, en Sonora se reportaron 220,368 casos de mujeres con al menos un incidente de violencia en el año 2003 (Instituto Nacional de Geografía e Informática, 2005). Otro estudio indica que, en México, el 70\% de las mujeres reporta haber sufrido algún tipo de violencia dentro del hogar (Violencia Doméstica, 2006). Una encuesta realizada por el Instituto Nacional de Geografía e Informática (INEGI, 2006) en el Distrito Federal señala que el $30 \%$ de las familias sufría algún tipo de violencia familiar como es el maltrato emocional, intimidación, abuso físico o sexual. El Informe Mundial sobre la Violencia y la Salud (Organización Panamericana de la Salud, 2003) indica que entre el 10\% y el $60 \%$ de las mujeres han sido objeto de agresiones físicas por parte de alguna pareja masculina en algún momento de su vida. Este mismo reporte muestra que aproximadamente la mitad de las mujeres habían sido víctimas de agresiones físicas por parte de su pareja.

Un estudio realizado en Sonora sobre maltrato infantil mostró que un $90 \%$ de las madres utilizaban castigo físico como método correctivo en la crianza de sus hijos (Frias \& McCloskey, 1998). La Procuraduría de la Defensa del Menor y la Familia del Estado de Sonora indicó que en el 2003 fueron reportadas 973; en 2004 fueron 954 y hasta el mes de julio de 2005 informaron de 1197 casos de maltrato infantil (G. Baldenebro Patrón, Procurador de la Defensa del Menor y la Familia, Comunicación personal, septiembre de 2005). En el caso del maltrato infantil, un $62 \%$ de las denuncias han sido comprobadas. En 2000 se produjeron 57,000 homicidios de niños menores de 15 años en el mundo (Organización Panamericana de la Salud, 2003). Estos datos nos muestran que la violencia en el hogar es un problema grave que ocurre muy frecuentemente en los hogares mexicanos y del mundo.

\section{Consecuencias de la violencia familiar}

La violencia familiar produce secuelas a corto y a largo plazo en los menores que son víctimas, entre las que se encuentran problemas en el aprovechamiento y en la conducta escolar (Dodge, Pettit, Bates, \& Valente, 1996; Shonk \& Ciccheti, 2001; Sternberg, Baradaran, Abbott, Lamb, \& Guterman, 2006). Otros estudios sugieren que el maltrato produce secuelas en la conducta interna y externa de los menores (Feiring, 2005; Sternberg, Lamb, Guterman \& Abbott, 2006; Zielinski \& Bradshaw, 2006) y problemas neurosicológicos y en las funciones cognitivas (Navalta, Polcari, Webster, Boghossian, \& Teicher, 2006). Los menores que son testigos de la violencia de los padres también presentan secuelas en su vida adulta (Davies, Dilillo, \& Martinez, 2004)

La conducta externa se refiere a un conjunto de problemas que manifiestan los niños en el ambiente externo (Jianghong, 2004). La literatura indica que esas conductas se manifiestan como hiperactividad, conducta disruptiva y agresividad
(Eisenberg et al., 2001). En cambio la conducta interna se refiere a problemas que afectan el ambiente psicológico del niño, como el estar cohibido, ansioso, deprimido (Campbell, Woods, Chouaf, \& Parker, 2000). Sin embargo, algunos autores reconocen que esta dicotonomía no es perfecta, y que existe comorbidad entre los problemas de conducta internos y externos (Jianghong, 2004).

La investigación en el área indica que los individuos que han sido abusados físicamente presentan más riesgo de problemas de conducta externa que los no abusados, tales como agresión (Lansford et al., 2002) y delincuencia (Stouthamer-Loeber, Loeber, Homish, \& Wei, 2001). Un estudio acerca de los problemas de conducta en niños encontró que los problemas de internalización se relacionaban con una pobre relación parental con los hijos, además de una baja competencia social, y un bajo estatus socio-económico de los padres. En cambio, los problemas de conducta externos se relacionaron con la baja competencia social y el bajo estatus socioeconómico de los padres, ser varón, experimentar disciplina severa, y una pobre supervisión (Lansford et al., 2006). Los niños maltratados exhibieron más sintomatología interna y externa que un grupo control de niños no maltratados, y los niños maltratados mostraron menos conductas pro-sociales adaptativas (Kim \& Cicchetti, 2004).

La experiencia de abuso en la niñez incrementa el riesgo para el daño social y psicológico (Margolin \& Gordis, 2000). Las víctimas de maltrato son más propensas a sufrir enfermedades y alteraciones anímicas y conductuales (depresión, ansiedad, problemas cognitivos, etc.) en la edad adulta (Kug, Dahlberg, Mercy, Zwi, \& Lozano, 2002) que los menores que no reciben maltrato. Los niños que han sido abusados se encuentran en grave riesgo de sufrir problemas de ajuste emocional como depresión y ansiedad (Johnson et al., 2002). Un estudio longitudinal y multi-informante llevado a cabo por Sternberg, Lamb, Guterman y Abbott (2006) reveló que la descripción de los problemas de conducta en las víctimas de abuso variaba dependiendo del informante y que las predicciones acerca de la relación entre abuso temprano y ajuste posterior fueron parcialmente confirmadas. Entrevistando a menores maltratados y no maltratados, los diferentes informantes no coincidieron totalmente en cuál de los grupos fue más severamente afectado. Este estudio también reveló que los niños eran los que variaban más la información dependiendo de la exposición actual a la violencia. Los niños que eran víctimas recientes reportaban más problemas de externalización y las niñas indicaron más problemas de internalización que los varones. Los niños jóvenes mostraron mayor susceptibilidad a los efectos de la violencia. Asimismo, se encontró una fuerte asociación entre la duración del abuso y daños en la memoria en mujeres que fueron víctimas de abuso sexual (Navalta et al., 2006). Además, los niños maltratados son menos populares en la escuela y menos aceptados entre sus compañeros que los no maltratados (Bolger \& Paterson, 2001).

La agresión hacia los niños en conjunción con un pobre funcionamiento familiar tiene un fuerte efecto en los problemas de consumo de alcohol en las víctimas (Engels, Vermulst, Dubas, Bot, \& Gerris, 2004). Los adolescentes que fueron abusados 
mostraron un frágil apego hacia sus padres en comparación con los que no fueron maltratados (Sternberg, Lamb, Guterman, Abbott, \& Dawud-Noursi, 2004). El maltrato infantil está asociado a altos niveles de ansiedad y depresión entre los jóvenes que han sido institucionalizados, además de que la experiencia del maltrato aumenta el riesgo de depresión en jóvenes que no han sido institucionalizados (Gover \& Layton-MacKenzie, 2003).

La violencia en la niñez aumenta el riesgo de que las víctimas utilicen el castigo físico en la edad adulta, independientemente de sus esquemas interpersonales (Merrill et al., 2005). Los estilos parentales tanto positivos como negativos también son transmitidos intergeneracionalmente (Belsky, Jaffee, Sligo, Wordward, \& Silva, 2005). El ser testigo de la violencia entre los padres se asocia a la depresión, baja autoestima y desorden post-traumático en los niños, aún después de controlar el abuso directo (Davies et al., 2004). Por otro lado, los altos niveles de apoyo materno y los bajos niveles de control rígido predicen altos niveles de simpatía, autovaloración y competencia social en los adolescentes (Laible \& Carlo, 2004). El cariño y el apoyo paterno siguen jugando un importante rol en el desarrollo socioemocional en la adolescencia aún cuando el soporte de los compañeros se vuelve sumamente importante (Laible, Carlo, \& Raffaelli, 2000). Los autores de estos estudios argumentan que el soporte y el control proveniente de los padres definen las competencias sociales, emocionales y morales de los adolescentes en muchas formas. Primera, los niños van a compartir los valores con los padres si sienten que éstos responden recíprocamente; segundo, los niños que tienen una relación positiva con sus padres adquieren orientaciones sociales positivas que pueden generalizar con otros. Finalmente, los niños adquieren importante información y habilidades sociales a partir de las interacciones con sus padres. Por lo tanto, podría decirse que el apoyo paterno se relaciona con una variedad de resultados positivos, como la alta autoestima, la conducta pro-social y bajos niveles de ansiedad, depresión y conducta agresiva (Laible et al., 2000). El control rígido de la conducta parece ser perjudicial para un ajuste saludable de los adolescentes (Laible \& Carlo, 2004). Hinton, Sheperies y Sims (2003), en una revisión de la literatura, encontraron que el soporte familiar era el predictor más importante para reducir la delincuencia juvenil.

Los niños maltratados manifiestan su comportamiento externo como conducta disruptiva o antisocial. Estos suelen ser niños que por lo general manifiestan bajo rendimiento académico, ausentismo, falta de autocontrol, conducta antisocial y quieren llamar la atención de sus compañeros y maestros en sus escuelas (Badia-Martín, 2005). La conducta antisocial se manifiesta con agresiones físicas, insultos, extorsión, vandalismo y intimidación, entre otros (Luiselli, Putnam, Handler, \& Feinberg, 2005).

La mayoría de estas investigaciones sobre las repercusiones del maltrato han medido las secuelas en los niños y muy pocas han utilizado otros informantes, aparte de los menores o sus padres, para evaluarlas. Por lo tanto, este trabajo de investigación pretende obtener información de los maestros de la escuela además de los reportes de los niños. Los datos fueron analizados mediante modelo de ecuaciones estructurales lo que posibilitó analizar al mismo tiempo el efecto de variables exógenas e intermedias en las variables dependientes.

Las victimas de violencia intrafamiliar presentan un riesgo muy grave de sufrir problemas emocionales como la depresión (Johnson et al., 2002). La literatura frecuentemente menciona que éstas van a exhibir problemas de ajuste emocional (Cicchetti \& Toth, 2000). Por lo tanto, se hipotetizó que la depresión pudiera ser una variable mediadora entre el maltrato y otros problemas de conducta.

Consecuentemente, el objetivo de la presente investigación fue analizar los problemas de ajuste emocional y de conducta en niños que han sido víctimas de maltrato, ya sea experimentada directamente o siendo testigos de episodios de violencia entre sus padres, a través del reporte de los profesores de las escuelas a las que asisten los menores.

\section{Método}

\section{Participantes}

De la muestra estudiada, $50(45.05 \%)$ niños pertenecen a la población general y $61(54.95 \%)$ son menores en situación de maltrato. Estos fueron niños que vivían en casas hogar porque sus padres fueron reportados ante las autoridades como maltratadores; por lo tanto los menores no vivían con sus padres en el momento del estudio. Las casas hogar son espacios habitacionales proporcionados por el gobierno para niños que son separados de sus progenitores. En estos espacios se les proporciona ayuda psicológica, económica y educativa, entre otras. La edad promedio de los participantes entrevistados fue de 10 años, con una mínima de 6 y máxima de 14 . El género de los entrevistados resultó ser de 84 menores (75.68\%) del sexo masculino y 27 (24.32\%) del sexo femenino. En promedio los participantes tenían 3 hermanos. La edad promedio del padre era de 33 años y la de la madre de 36 años. El grado escolar de los menores oscilaba entre el primero y el sexto de primaria. La mayoría de ellos cursaba el cuarto (29\%) y el sexto grado (27\%) escolar. La mayoría de las madres eran solteras o vivían en unión libre. Únicamente el 12\% de ellas estaban casadas. El 60\% de la madres se dedicaba al hogar y aproximadamente el 70\% de los padres eran empleados. En su mayoría los progenitores habían cursado hasta la enseñanza preparatoria $(86 \%$ de los padres y $60 \%$ de las madres).

\section{Instrumentos}

El estudio utilizó dos baterías de instrumentos. Una fue aplicada a los maestros y otra a los menores. Se obtuvieron variables demográficas tales como la ocupación, el nivel escolar y el ingreso de los padres, así como el nivel educativo de los menores. A los niños se les aplicó la Escala de Tácticas de Conflictos de Straus, Hamby, Finkelhor, Moore y Runyan (1998), la cual mide el grado de violencia que recibían los menores por parte de su madre y padre (maltrato infantil) y la violencia que manifiestan los padres entre sí (violencia en la pareja). Asimismo, se les administró la escala de depresión de Hamilton (1959). A los maestros se les aplicó la lista de Chequeo de Achenbach (2005, Licencia \# 154-6-13-05). 


\section{Variables}

Maltrato o castigo. Esta variable fue medida a través de la Escala de Tácticas de Conflicto de Straus et al. (1998). Esta escala fue elaborada en inglés y traducida al español por expertos y vuelta a traducir al inglés, con el fin de confirmar su correcta traducción. La escala ha sido utilizada en otros estudios en poblaciones mexicanas mostrando un alfa de Cronbach de .70 (Frías, Corral, Moreno, \& Rodríguez, 2000; Frías, Ramírez, Soto, Castell, \& Corral, 2000). El instrumento contiene reactivos que miden la frecuencia de la violencia que ejercen los padres hacia los hijos en una escala de cero a seis, en donde: $0=$ nunca, $1=$ una vez, 2 =dos veces, $3=$ tres a cinco veces, $4=$ de seis a diez veces, $5=$ de once a veinte veces y $6=$ más de veinte veces. Straus (1991) reportó un alfa de Cronbach de .72 para toda la escala.

Violencia hacia las mujeres. La violencia hacia las mujeres fue medida con la escala de Tácticas de Conflicto de Straus (1990). Esta escala al igual que la anterior fue elaborada en los Estados Unidos y traducida al español por expertos. Los reactivos del cuestionario miden la frecuencia con la que las mujeres recibieron agresiones por parte de sus parejas en un rango de cero a seis, en donde: $0=$ nunca, $1=$ una vez, $2=$ dos veces, $3=$ tres a cinco veces, $4=$ de seis a diez veces, $5=$ de once a veinte veces y $6=$ más de veinte veces. Los niños contestaban cuantas veces observaban esas conductas en sus padres. Para eso, el cuestionario fue modificado para que los niños lo contestaran y piloteado nuevamente. Straus reportó un alfa de Cronbach de .78 para toda la escala. El alfa que se obtuvo en este estudio fue de .92 .

Depresión. Se utilizaron reactivos de la Escala de Depresión de Hamilton (1959). Los menores reportaron el número de veces que presentaron alguno de los síntomas considerados, en las últimas dos semanas, empleando una escala de 0 a 4 , en donde 0 = ningún día, 1 = uno o dos días, 2 = de tres a cinco días, 3 = de seis a diez días y 4 = más de diez días. El instrumento descrito ha sido empleado en estudios previos desarrollados en la localidad; Castell, Frías, Corral y Sotomayor (2000) reportaron un alfa de Cronbach de .69; Figueredo et al. (2001) obtuvieron una alfa de .79 y Frías (2002) reportó una alfa de .72. En este estudio, con la adaptación que se llevó a cabo para los menores, se obtuvo un alfa de .84 .

Problemas en los niños. Para medir los problemas sociales y de conducta en los niños se utilizó la Lista de Chequeo para profesores de Achenbach (Achenbach, 1991, Achenbach \& McConaughy, 1997). Esta escala contiene 117 preguntas que describen problemas emocionales o de conducta de los niños. Cada reactivo mide en forma cuantitativa la ocurrencia de esos problemas de conducta en los últimos seis meses en una escala de 0 a 2, en donde: $(0=$ no es cierto, $1=$ en cierta manera, $2=$ muy cierto). Los aspectos que mide el instrumento son el retraimiento, problemas psicosomáticos, ansiedad, depresión, problemas sociales, problemas de atención, conducta delictiva, agresividad, problemas de pensamiento y otros problemas conductuales. Las respuestas de cada uno de los reactivos fueron promediadas para formar cada uno de los índices descritos anteriormente.

\section{Procedimiento}

La muestra de menores maltratados fue obtenida de los registros de la casa hogar donde residían. Todos los menores que se encontraban en la casa hogar que tenían menos de un mes de ingreso fueron seleccionados. Los de la población en general se seleccionaron equiparando las características demográficas (edad, escolaridad y sexo) con los niños de los padres reportados como maltratadores. Primeramente se obtuvo el consentimiento de los padres de los menores que participaron en el estudio. Los niños en situación de maltrato fueron entrevistados en la casa hogar en donde residían. La dirección de las escuelas a las que asistían los menores internos fue solicitada a las autoridades de la casa hogar. Los menores de población general fueron entrevistados en las escuelas a donde asistían. A todos se les pidió su participación voluntaria, y se les aseguró la confidencialidad de la información proporcionada; además fueron notificados de que podían dejar de contestar en cualquier momento si alguna pregunta no les parecía conveniente o les incomodaba. Los menores y los profesores fueron entrevistados por estudiantes de psicología educativa. La entrevista duró alrededor de 20 minutos para los niños y 30 minutos aproximadamente para los profesores.

\section{Análisis de datos}

Se obtuvieron estadísticas univariadas, medias y desviaciones estándar para las variables continuas y frecuencias para las variables categóricas. Además, se calcularon alfas de Cronbach para estimar la confiabilidad de las escalas. Con el fin de agrupar las variables se elaboraron índices con los reactivos de las escalas incluidas en el estudio. Un índice se conforma promediando las respuestas de todas las variables dentro de una escala. Se presume la representatividad del índice después de que se obtiene la consistencia de ésta, según lo indica el alfa de Cronbach. Se formaron 7 índices, representando la violencia entre pareja, el maltrato recibido, la depresión, la conducta delictiva, problemas de pensamiento, ansiedad y retraimiento. Otras variables propuestas por Achenbach (Achenbach \& McConaughy, 1997) como son los problemas sociales, la agresividad y los problemas de atención no fueron incluidas en el modelo porque estaban altamente correlacionadas con el factor de conducta delictiva y producían una gran colinearidad.

Se especificó un modelo de ecuaciones estructurales en el que se estimó el efecto directo de la variable independiente (la violencia familiar) en la variable mediadora depresión, y los de ésta en la variable dependiente problemas de conducta. Los datos de ambos grupos fueron unidos para obtener mayor variabilidad en el análisis, ya que se encontró que los dos grupos experimentaban algún tipo de maltrato.

El análisis de ecuaciones estructurales contiene dos componentes principales: el modelo de medición y el modelo estructural. El modelo de medición consiste en un análisis factorial confirmatorio en donde las variables latentes se conforman con las variables observadas (Bentler, 2006). Este estudio hipotetizó la presencia de dos factores: el de violencia familiar, formado por los índices maltrato infantil y violencia hacia la mujer; y el factor de problemas de conducta, con los índices de conducta delictiva, problemas de pensamiento, ansiedad y retraimiento. El modelo estructural se refiere a las relaciones entre las variables latentes o factores formados, y entre 
éstos y las variables observadas. En este modelo se hipotetizó que el maltrato infantil afectaría a las variables afectivas de los alumnos y éstas a los problemas de conducta de los menores.

Para determinar la pertinencia del modelo propuesto se utilizaron indicadores de bondad de ajuste. La bondad de ajuste es un indicador de la pertinencia de una teoría de relaciones entre variables, dada por la correspondencia entre el modelo que la representa y los datos utilizados para probar esa teoría (Corral, Frías, \& González, 2001). Para medir la bondad de ajuste del modelo se utilizaron dos indicadores: el indicador estadístico $C h i$ cuadrada $\left(\chi^{2}\right)$ que determina la diferencia entre el modelo teórico propuesto y un modelo saturado formado por las relaciones entre todas las variables. Si el modelo teórico es pertinente, éste no es diferente del saturado, por lo que la $\chi^{2}$ tendrá un valor bajo y no significativo $(p>.05)$. Otros estadísticos empleados son los indicadores prácticos que se derivan de la $\chi^{2}$; éstos controlan el efecto del número de sujetos sobre la significatividad de la comparación. El nivel mínimo aceptable de los indicadores prácticos para considerar un modelo con buena bondad de ajuste es de .90. Estos indicadores son el Índice Bentler-Bonett de Ajuste No Normado (NNFI, por sus siglas en inglés) y el Índice de Ajuste Comparativo ( $C F I$ ). También se consideró el indicador de la Raíz Cuadrada del Cuadrado Medio del Error de Aproximación ( $R M S E A$, por sus siglas en inglés) cuyo valor deberá ser menor a .08 como evidencia de bondad de ajuste (Browne \& Cudeck, 1993).

\section{Resultados}

La Tabla 1 muestra el reporte de los menores respecto del maltrato que recibieron. Un $68 \%$ de los niños reportó haber sido agredido por su madre al menos una vez en los últimos 6 meses y un $60 \%$ por su padre; el $20 \%$ indicó haber recibido formas severas de abuso como patadas y aventones por sus padres en ese mismo período.

Tabla 1

Porcentaje del abuso que reciben los menores al menos una vez en los últimos 6 meses

\begin{tabular}{lcc}
\hline \multirow{2}{*}{ Indicador de abuso } & \multicolumn{2}{c}{ Porcentaje } \\
\cline { 2 - 3 } & Padre & Madre \\
\hline Te quita privilegio & 50 & 58 \\
Te manda a tu cuarto & 37.5 & 42 \\
Te grita & 60 & 68 \\
Te amenaza con pegarte & 49 & 65 \\
Te dice groserías & 37 & 48 \\
Te llama tonto, flojo & 41 & 49 \\
Te dice que te correrá & 16 & 20 \\
Te correrá a golpes & 21 & 15 \\
Te golpea con la mano & 53 & 61 \\
Te pega en la mano, brazo & 45 & 50 \\
Te pega con un cinturón & 50 & 53 \\
Te pega en la cara & 25 & 29 \\
Te da patadas & 15 & 16 \\
Te jala o avienta & 21 & 22 \\
Te pega durante unos min. & 20 & 19 \\
\hline
\end{tabular}

La Tabla 2 muestra el porcentaje de los episodios de violencia entre sus padres presenciados por los menores. Esta tabla indica el porcentaje con la que las madres sufren violencia de parte de sus parejas; más del $65 \%$ de las mujeres han sido víctimas de algún tipo de agresión en los últimos 6 meses.

Tabla 2

Porcentaje de los episodios de violencia familiar presenciados por el hijo(a), reportado por el hijo(a)

\begin{tabular}{lc}
\hline Indicador de maltrato & Porcentaje \\
\hline Insulta o dice malas palabras & 65 \\
Le tira con algún objeto & 50 \\
La empujan & 30 \\
Le pega cachetadas & 36 \\
Le pega patadas & 34 \\
La golpea & 17 \\
La ha tratado de estrangular & 13 \\
La ha acuchillado & 26 \\
Le ha roto ropa & 8 \\
\hline
\end{tabular}

La Tabla 3 muestra las alfas de Cronbach de las escalas utilizadas en el modelo. Todas ellas resultaron mayores a .60 lo cual es considerado aceptable (Nieva \& Sorra, 2003).

Tabla 3.

Alfas de Cronbach para las escalas del estudio

\begin{tabular}{lc}
\hline Escala/Variables & Alfa \\
\hline Violencia hacia las mujeres & .92 \\
Maltrato & .83 \\
Depresión & .84 \\
Conducta delictiva & .79 \\
Problemas de pensamiento & .73 \\
Retraimiento & .66 \\
Ansiedad & .86 \\
\hline
\end{tabular}

La Figura 1 muestra los resultados del modelo estructural. Primeramente se probó la validez de constructo de las variables latentes (violencia familiar y problemas de conducta en niños). Este modelo produjo pesos factoriales altos y significativos en todos los indicadores, los cuales indican validez convergente y los valores más bajos de covarianza entre los factores indica validez discriminante (Corral \& Figueredo, 1999).

El modelo de medición mostró que los dos índices del factor Violencia Familiar (maltrato al niño y violencia hacia la mujer) obtuvieron un peso factorial de .77. El índice de conducta delictiva tuvo un peso factorial de .87 , el índice de problemas de pensamiento produjo una carga factorial de .92 , el índice de retraimiento obtuvo un peso factorial de $.69 \mathrm{y}$ el de ansiedad fue de .77 sobre el factor de problemas de comportamiento. Las correlaciones altas y significativas entre los indicadores y su correspondiente factor fueron tomadas como evidencia de validez de constructo de los modelos de medición (Corral et al., 2001). En el modelo estructural la violencia familiar produjo un efecto directo y significativo en la depresión (coeficiente estructural .31) y ésta en los problemas de comportamiento en 
los niños (coeficiente estructural .16). Por lo tanto, la violencia familiar ejerció un efecto indirecto en los problemas de comportamiento de los menores.

Los indicadores de bondad de ajuste para el modelo probado fueron aceptables. La probabilidad del indicador estadístico $\chi^{2}$ fue mayor a .05; y los indicadores prácticos rebasaron el corte establecido de $>.90$ y el valor de RMSEA fue de .04 . La $R^{2}$ del modelo fue de .15 lo cual nos indica que predice el $15 \%$ de la varianza total en los problemas de comportamiento de los niños.

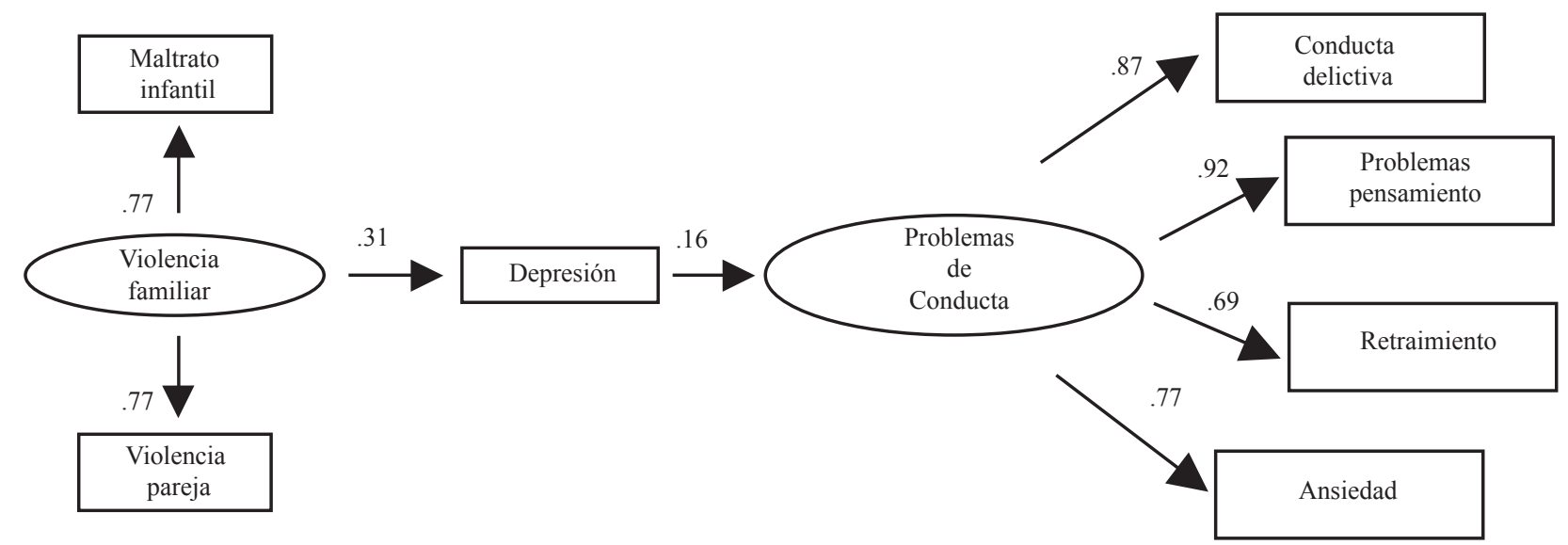

Figura 1. Modelo estructural de violencia familiar como predictor de problemas del comportamiento. $\chi^{2}=42.66(13 \mathrm{gl}), p=.001 ; N N F I=.85 ; C F I=.91 ; \mathrm{RMSEA}=.042 ; R^{2}=.15$.

\section{Discusión}

Los resultados del estudio muestran que la violencia familiar en sus dos formas directa (maltrato infantil) e indirecta (cuando son testigos de la violencia entre sus padres) producen problemas emocionales y de comportamiento en sus víctimas. La violencia familiar tuvo un efecto positivo en la depresión, lo cual indica que entre más violencia experimenten los menores más depresión manifestarán. Coincidiendo con otros estudios, las víctimas de maltrato manifiestan más problemas emocionales que los que no son abusados y una de estas manifestaciones es la depresión (Organización Panamericana de la Salud, 2003; Johnson et al., 2002). Las interacciones negativas con los padres ocasionan que los menores sufran de esta alteración anímica (Lansford et al., 2006). La literatura igualmente menciona que el maltrato produce problemas de ajuste emocional, que se manifiestan en depresión y ansiedad (Davies et al., 2004; Éthier, Lemelin, \& Lacharité, 2004; Kaplow \& Widom, 2007).

En el mismo sentido, el efecto positivo de la depresión en los problemas de conducta indica que entre más depresión desarrollen, más problemas de conducta manifestarán los menores. El modelo también muestra que las víctimas de maltrato exhiben problemas de conducta en el ámbito escolar, como son la antisocialidad, la falta de concentración y la ansiedad. Esto concuerda con los estudios realizados por Dodge et al. (1996); Shonk y Ciccheti (2001); Sternberg, Baradaran, et al. (2006) en los cuales se reporta que los menores que fueron víctimas de abuso manifiestan problemas de aprovechamiento y conducta escolar. De la misma manera, numerosos estudios (Kim \& Cicchetti, 2004; Lansford et al., 2002; StouthamerLoeber, Loeber, Homish, \& Wei, 2001) han mostrado que los menores maltratados manifiestan más conducta antisocial en la adolescencia y delincuencia en la edad adulta. En cambio, los niños que tienen una buena relación con sus padres adquieren orientaciones pro-sociales que se manifiestan en el ámbito escolar. La conducta antisocial es la que se encuentra en contra de las normas sociales, en cambio la delictiva es la que está en contra de las normas legales. Estos dos tipos de conducta en algunos casos son equiparables: la única diferencia es que la que se considera delito es la que está establecida por la ley como tal. En el presente estudio el constructo de conducta delictiva fue elaborado de acuerdo con la operacionalización de Achenbach y McConaughy (1997), que considera como delictivas las formas más severas de conducta antisocial.

Nuestros resultados indican que el maltrato produce problemas en el ajuste emocional, social y conductual en los niños y que éstos se pueden manifestar en la escuela, en el hogar o en la comunidad en donde viven. Los problemas de ajuste les impiden desarrollarse en el ámbito escolar. Los niños maltratados pueden exhibir conductas de retraimiento, de incomunicación, de encierro o disruptivas. Las tres primeras se consideran de internalización y la última de externalización.

El modelo predijo únicamente el $15 \%$ de la varianza; esto pudiera deberse a que en este modelo se combinaron los reportes de los maestros y los de los menores. Los reportes de depresión y violencia fueron llevados a cabo por los niños, mientras que los de problemas de conducta fueron elaborados por los maestros. Los dos reportes pudieran no concordar en lo informado, tal como lo sugieren los estudios de Sternberg, Lamb, et al. (2006). Estos autores indican que cuando se utilizan varios informantes los resultados no coinciden, y los reportes de maestros difieren más al de los niños que los reportes de las madres con el de sus hijos. Es probable que, aunque los maestros pudieran percibir los problemas de conducta de los niños, ellos no están cabalmente enterados de su problemática o, al menos, no tanto como los padres. Los maestros reportan principalmente los problemas de conducta externa y los niños los de conducta interna. Sin embargo, este mismo autor menciona que las futuras investigaciones tienen que considerar la obtención de información de varias fuentes ya 
que esta aportaría una perspectiva más válida acerca de cómo es el funcionamiento de los niños. Otro aspecto importante que se debe considerar en la evaluación de los niños es la percepción que tienen ellos sobre sí mismos (Sternberg, Baradaran, et al., 2006). La utilización de múltiples informantes pudiera ayudar a un mejor entendimiento de los efectos de la violencia familiar, proporcionando la percepción externa de los padres y de los maestros y la interna de los niños. Por otro lado esta perspectiva tiene implicaciones para la planeación y evaluación de intervenciones, ya que esta atendería todos los problemas que se presentan en las víctimas de abuso.

\section{Conclusiones}

Los maestros manifestaron que los alumnos exteriorizaban muchos problemas en el aula. Los datos nos indican que los maestros de la escuela perciben los problemas de conducta de los menores y que estos se manifiestan en el ámbito escolar. Consecuentemente, es necesario entrenar a los maestros en el manejo de esta problemática pues ellos pudieran solucionar parte de los problemas que se presentan en el aula y ayudarían, de alguna manera, a los menores en el desempeño en la escuela y con esto mejoraría el aprovechamiento escolar. Además, lo expuesto por nuestro estudio muestra la necesidad de utilizar múltiples informantes para la obtención de datos. En este caso el reporte del maestro de escuela es una fuente adicional de información que nos indica cómo el menor funciona en la escuela, además del ámbito familiar. Mediante el reporte de los maestros, esta investigación nos indicó que los menores manifiestan conductas problemáticas desde la edad escolar, pudiendo ser detectadas por sus profesores. Los resultados de los reportes obtenidos ayudarían a crear programas de detección temprana del maltrato infantil en las escuelas con el fin de atender a los menores lo más pronto posible, dentro y fuera del aula, y así evitar que se generen problemas graves como la deserción escolar y la delincuencia.

\section{Referencias}

Achenbach, T. M. (1991). Integrative guide for the CBCL/4-18 yrs, TRF profiles. Burlington: University of Vermont, Department of Psychiatry.

Achenbach, T. M. (2005). Advancing assessment of children and adolescents: commentary on evidence-based assessment of child and adolescents disorders. Journal of Clinical Child and Adolescent Psychology, 34(3), 541-547.

Achenbach, T. M., \& McConaughy, S. H. (1997). Empirically based assessment of child and adolescent psychopathology. Thousand Oaks, California: Sage.

Badia-Martín, M. M. (2005). Gravedad e importancia de los comportamientos de indisciplina. Causas y factores asociados a ciertas conductas: Una revisión de ciertos estudios. Psicología Educativa, 11(2), 65-78.

Belsky, J., Jaffee, S. R., Sligo, J., Wordward, L., \& Silva, P. A. (2005). Intergenerational transmission of warm-sensitive-stimulating parenting: A prospective study of mothers and fathers of 3 years old. Child Development, 2, 384-396.

Bentler, P. M. (2006). EQS, Structural Equation Program Manual. Los Angeles: BMDP Statistical Software.

Bolger, K. E., \& Paterson, C. J. (2001). Developmental pathways from child maltreatment to peer rejection. Child Development, 72, 549-568.

Browne, M. W., \& Cudeck, R. (1993). Alternative ways of assessing model fit. In
K. A. Bollen \& J. S. Long (Orgs.), Testing structural equation models (pp. 136-162). Thousand Oaks, California: Sage.

Campbell, J. C., Woods, A. B., Chouaf, K. L., \& Parker, B. (2000). Reproductive health consequences of intimate partner violence: A nursing research review. Clinical Nursing Research, 9, 217-237.

Castell, I., Frías, M., Corral V., \& Sotomayor, M. (2000). Apoyo familiar, castigo corporal y sus repercusiones conductuales en niños. Revista Mexicana de Psicología, 17(1), 37-45.

Cicchetti, D., \& Toth, S. L. (2000). Developmental processes in maltreated children. Nebraska Symposium on Motivation, 46, 85-160.

Corral, V., \& Figueredo, A. J. (1999). Convergent and divergent validity of three measures of conservation behavior: The multitrait-multimethod approach. Enviroment \& Behavior, 31, 805-820.

Corral, V., Frías, M., \& González, D. (2001). Análisis cuantitativos de variables latentes. Hermosillo, México: UniSon.

Davies, C. A., Dilillo, D., \& Martinez, I. G., (2004). Isolating adult psychological correlates of witnesing parental violence: Findings from predominantly Latina sample. Journal of Family Violence, 19, 377-385.

Dodge, K. A., Pettit, G. S., Bates, J. E., \& Valente, E. (1996). Social information processing patterns partially mediate the effect of early physical abuse on later conduct problems. Journal of Abnormal Psychology, 104, 632-643.

Eisenberg, N., Cumberland, A., Spinrad, T. L., Fabes, R. A., Shepard, S. A., Reiser, M., Murphy, B. C., Losoya, S. H., \& Guthrie, I. K. (2001). The relations of regulation and emotionality to children's externalizing and internalizing problem behavior. Child Development, 72, 1112-1134.

Engels, R. C. M. E., Vermulst, E. E., Dubas, J. S., Bot, S. M., \& Gerris, J. (2004) Long term effects of family functioning and child characteristics on problem drinking in young adulthood. European Addiction Research, 11, 32-37.

Éthier, L. S, Lemelin, J. P., \& Lacharitè, C. (2004). A longitudinal study of the effects of chronic maltreatment on children's behavioral and emotional problems. Child Abuse and Neglect, 28, 1265-1278.

Feiring, C. (2005). Emotional development, shame, and adaptation to child maltreatment. Child Maltreatment, 10(4), 307-310.

Figueredo, J. A., Corral, V., Frías, M., Bachar, K. J., White, J, McNeill, P. L., Kirsner, B. R., \& Castell, R. I. (2001). Blood, solidarity, status and honor: The sexual balance of power and spousal abuse in Sonora, Mexico. Evolution and Human Behavior, 22, 295-328.

Frías, M. (2002). Long- term effects of child punishment on Mexican women: A structural model. Child Abuse and Neglect, 26, 371-386.

Frías, M., Corral, V., Moreno, C., \& Rodríguez, I. (2000). El Maltrato infantil como un factor de riesgo para la salud, la conducta pro social y la ejecución escolar en niñas y niños. In E. Ramos (Org.), Investigaciones Educativas en Sonora (Vol. 2, pp.14-31) Hermosillo, México: REDIES.

Frías, M., \& McCloskey, L. A. (1998). Determinants of harsh parenting in México. Journal of Abnormal Child Psychology, 26, 129-139.

Frías, M., Ramírez, J. M., Soto, R., Castell, I., \& Corral, V. (2000). Repercusiones del maltrato infantil: un estudio con niños en alto riesgo de maltrato. In Asociación Mexicana de Psicología Social-AMEPSO (Org.), La Psicología Social en México (Vol. 8, pp. 479-485). México: Autor.

Gover, D., \& Layton-MacKenzie, A. R. (2003). Child maltreatment and adjustment to juvenile correctional institutions. Criminal Justice and Behavior, 30, 374-396.

Hamilton, M. (1959). The assessment of anxiety states by ratings. British Journal of Medical Psychology, 32, 50-55.

Hinton, W. J., Sheperies, C., \& Sims, P. (2003). Family based approach to juvenile delinquency: a review of the literature. The Family Journal: Counseling and Therapy for Couples and Families, 11, 167-173.

Instituto Nacional de Geografía e Informática - INEGI (2005). Recuperado el 16 de octubre de 2005, de http://www.inegi.gob.mx.

Instituto Nacional de Geografía e Informática - INEGI (2006). Recuperado el 15 de mayo de 2006, de http://www.inegi.gob.mx/est/contenidos/espanol/ 
rutinas/ept.asp?t $=$ mvio33\&c $=5521$.

Jianghong, L. (2004). Childhood externalizing behavior: Theory and implications. Journal of Child and Psychiatric Nursing, 7. Recuperado el 15 de mayo, 2006, de http://findarticles.com/p/articles/mi_qa3892/is_200407/ai_n9457065.

Johnson, R. M., Kotch, J. B., Catellier, D. J., Winsor, J. R., Dufort, V., Hunter, W., \& Amaya-Jackson, L. (2002). Adverse behavioral and emotional outcomes from child abuse and witnessed violence. Child Maltreatment, 7, 179-186.

Kaplow, J. B., \& Widom, C. P. (2007). Age of onset of child maltreatment predicts long-term mental health outcomes. Journal of Abnormal Psychology, 116(1), 176-187.

Kim, J., \& Cicchetti, D. (2004). A longitudinal study of child maltreatment, mother child relationship quality and maladjustment: the role of self-esteem and social competence. Journal of Abnormal Child Psychology, 32, 341-354.

Kug, E. G., Dahlberg, L. L., Mercy, J. A., Zwi, A. B., \& Lozano, R. (2002). World Report on Violence and Health (WHO). Recuperado el 8 de marzo de 2003, de http://www.who.int/violence_injury_prevention/violence/world_report/ en/full_en.pdf.

Laible, D. J., \& Carlo, G. (2004). The differential relations of maternal and paternal support and control to adolescent social competence, self-worth, sympathy. Journal of Adolescent Research, 19, 759-782.

Laible, D. J., Carlo, G., \& Raffaelli, M. (2000). The differential impact of parent and peer attachment on adolescent adjustment. Journal of Youth and Adolescence, 29, 45-59.

Lansford, J. E., Dodge, K. A., Pettit, G. S., Bates, J. E., Crozier, J., \& Kaplow, J. (2002). A 12- year prospective study of the long-term effects of early child physical maltreatment on psychological, behavioral, and academic problems in adolescence. Archives of Pediatrics and Adolescent Medicine, $156,824-830$.

Lansford, J. E., Malone, P. S., Stevens, K. I. Dodge, K. A., Bates, J. E., \& Pettit, G. S. (2006). Developmental trajectories of externalizing and internalizing behavior: factors underlying resilience in physically abused children. Development and Psychopathology, 18, 35-55.

Luiselli, J. K., Putnam, R. F., Handler, M. W., \& Feinberg, A. B. (2005). Wholeschool positive behavior support: effects on student discipline problems and academic performance. Educational Psychology, 25, 183-198.

Margolin, G., \& Gordis, E. B. (2000). The effects of family and community violence on children. Annual Review of Psychology, 51, 445-479.

Merrill, L. L., Thomsen, C. J., Crouch, J. L., May, P., Gold, S. L., \& Milner, J. S. (2005). Predicting adult risk of physical child abuse from childhood exposure to violence: can interpersonal schemata explain the association. Journal of Social and Clinical Psychology, 24, 981-1002.

Navalta, C. P., Polcari, A., Webster, D. M., Boghossian, A., \& Teicher, M. H. (2006). Effects of childhood sexual abuse on neuropsychological and cognitive function in college women. Journal of Neuropsychiatry Clinical Neurosciences, 18, 45-53.
Nieva, V. F., \& Sorra, J. (2003). Safety culture assessment: a tool for improving patient safety in healthcare organizations. Quality Safe Health Care, 12 (supl), ii17-ii23.

Organización Panamericana de la Salud. (2003). Informe Mundial sobre la Violencia y la Salud. Washington, DC: Oficina Regional para las Américas de la Organización Mundial de la Salud. Recuperado el 8 de marzo de 2003, de http://www.paho.org/Spanish/AM/PUB/Contenido.pdf.

Shonk, S., \& Ciccheti, D. (2001). Maltreatment, competency deficits, and risk for academic and behavioral maladjustment. Developmental Psychology, 37, 3-17.

Sternberg, K. J., Baradaran, L. P., Abbott, C. B., Lamb, M. E., \& Guterman, E. (2006). Type of violence, age, and gender differences in the effects of family violence on children's behavior problems: a meta-analysis. Developmental Review, 26, 89-112.

Sternberg, K. J., Lamb, M. E., Guterman, E., \& Abbott, C. B. (2006). Effects of early and family violence on children's behavior problems and depression: a longitudinal, multi-informant perspective. Child Abuse \& Neglect, 30, 283-306.

Sternberg, K. J., Lamb, M. E., Guterman, E., Abbott, C. B., \& Dawud-Noursi, S. (2004). Adolescents' perceptions of attachments to their mothers and fathers in families with histories of domestic violence: a longitudinal perspective. Child Abuse \& Neglect, 29, 853-869.

Stouthamer-Loeber, M., Loeber, R., Homish, D. L., \& Wei, E. (2001). Maltreatment of boys and the development of disruptive and delinquent behavior. Development and Psychopatology, 13, 941-955.

Straus, M. A. (1990). The Conflict Tactics Scales and its critics: an evaluation and new data on validity and reliability. In M. A. Straus \& R. J. Gelles (Orgs.), Physical violence in American families: risk factors and adaptations to violence in 8,145 families (pp. 49-73). New Brunswick, New Jersey: Transaction Publishing.

Straus, M. (1991). Discipline and deviance: physical punishment of children and violence in adulthood. Durham: University of New Hampshire, Family Research Laboratory.

Straus, M., Hamby Sh., Finkelhor, D., Moore, D., \& Runyan, D. (1998). Identification of child maltreatment with the parent-child conflict tactics scales: development and psychometric data for a national sample of American parents. Child Abuse \& Neglect, 22(4), 249-270.

Violencia domestica. (2006). Recuperado el 15 de mayo de 2006, de http://www. arte-sana.com/espanol_statistics2.htm.

World Health Organization - WHO (1999). Report of the Consultation on Child Abuse Prevention, Geneva. Recuperado el 8 de marzo de 2003, de http:// www.anppcan.org/anppcan/deftext.htm.

Zielinski, D. S., \& Bradshaw, C. P. (2006). Ecological influences on the sequelae of child maltreatment: a review of the literature. Child Maltreatment, 11, 49-62. 
${ }^{1}$ Este estudio se realizó gracias al apoyo fondo Mixto CONACYT-Gobierno del Estado de Sonora (Fondos mixtos, convenio son-2004-C02-13).

Martha Frías Armenta, doutora em Psicologia pela Universidade do Arizona (EUA), é professora na Universidade de Sonora (México). Endereço para correspondência: Martha Frías Armenta, Sevilla \#6, Fracc. Casa Grande - Sección III; Hermosillo, Sonora; 83246, México. E-mail: marthafrias@sociales.uson.mx

Blanca Fraijo Sing, doutora em Ciências Sociais pela Universidade Autônoma de Sinaloa (México), é professora na Universidade de Sonora (México).

Nehemias Cuamba Osorio, é aluno de Licenciatura em Psicologia, Universidade de Sonora (México). 